\section{Shift of activity from attention to motor-related brain areas during visual learning}

\author{
Stefan Pollmann ${ }^{1,2} \&$ Marianne Maertens ${ }^{1-3}$ \\ With practice, we become increasingly efficient at visual object \\ comparisons. This may be due to the formation of a memory \\ template that not only binds individual features together to \\ create an object, but also links the object with an associated \\ response. In a longitudinal fMRI study of object matching, \\ evidence for this link between perception and action was \\ observed as a shift of activation from visual-attentive \\ processing areas along the posterior intraparietal sulcus \\ to hand-sensory and motor-related areas.
}

When we perceive an object, we often link a specific response to it. For instance, perception of a red traffic light primes a movement of the car driver's foot onto the brake pedal. Thus, object perception does not only involve perceptual processes but may have direct effects on the selection of appropriate actions ${ }^{1}$. In this example, the perception of a red traffic light not only activates an object representation but also creates a link to the motor actions required for braking. Of course, we do not automatically brake whenever we see a red traffic light, but the link between object representation and response facilitates braking when we do decide to execute this action.

In a longitudinal fMRI study, we tested this hypothesis of a link between perception and response by investigating learning-related

Figure 1 Stimuli and procedures. Top, the stimulus set consisted of five categories of geometric shapes: square, triangle, parallelogram, trapezoid and hexagon. Each category consisted of two exemplars that were identical in form but different in orientation. Four items were presented in a trial, two on each side of fixation. In the physical-identity (PI) matching task, a difference in orientation between otherwise identical shapes precluded a 'match' judgment. In the category-identity $(\mathrm{Cl})$ matching task, participants had to match category membership (for example, 'triangle', 'hexagon') irrespective of orientation. Bottom, a trial began with the presentation of a fixation cross for either $100 \mathrm{~ms}$ or $600 \mathrm{~ms}$. Next, two red frames appeared for $100 \mathrm{~ms}$ and cued the locations of two of the four items to be matched. The cues were followed by the presentation of four geometric shapes appearing simultaneously at the four display locations for $80 \mathrm{~ms}$. Participants had to indicate match or mismatch by pressing one of two buttons with their index finger or middle finger, respectively. Response time registration began with the onset of the target display and ended with the participant's response or after a maximum of $2,220 \mathrm{~ms}$ or $2,720 \mathrm{~ms}$, for a total trial duration of $3,000 \mathrm{~ms}$. Participants received a $2,000-\mathrm{Hz}$ feedback tone after every incorrect response. See Supplementary Methods for further details. changes in brain activation during visual object-matching. In this task, the attentional demands for analyzing feature differences between two objects are initially high, but they should decrease with learning when a memory template of the object pair is created ${ }^{2}$. In turn, strengthening the memory template may strengthen the link to the associated response $\mathrm{e}^{1,3}$. In terms of neural activation, we expect a decrease over the course of learning in brain areas that support attentive processing of stimulus features, whereas we expect an increase in areas that link visual input to manual response preparation.

Attentive visual processing depends on posterior parietal cortex, as indicated, for example, by deficits in attentionally demanding visual search performance after temporary disruption of parietal function by transcranial magnetic stimulation (TMS) ${ }^{4}$. In particular, the cortex along the horizontal segment ${ }^{5}$ of the intraparietal sulcus (IPS) is involved in shifting attention in space ${ }^{6,7}$ or between feature dimensions $^{8}$. Disruption of attentionally demanding visual search by parietal TMS disappears when search becomes automatic after learning ${ }^{4}$. Similarly, activation in the superior parietal lobule decreases after skill learning 9 . In contrast, activity related to the representation of conditional manual responses would be expected to occur in the hand motor area and in dorsal premotor cortex, which supports conditional responses associated with arbitrary stimuli ${ }^{10}$.
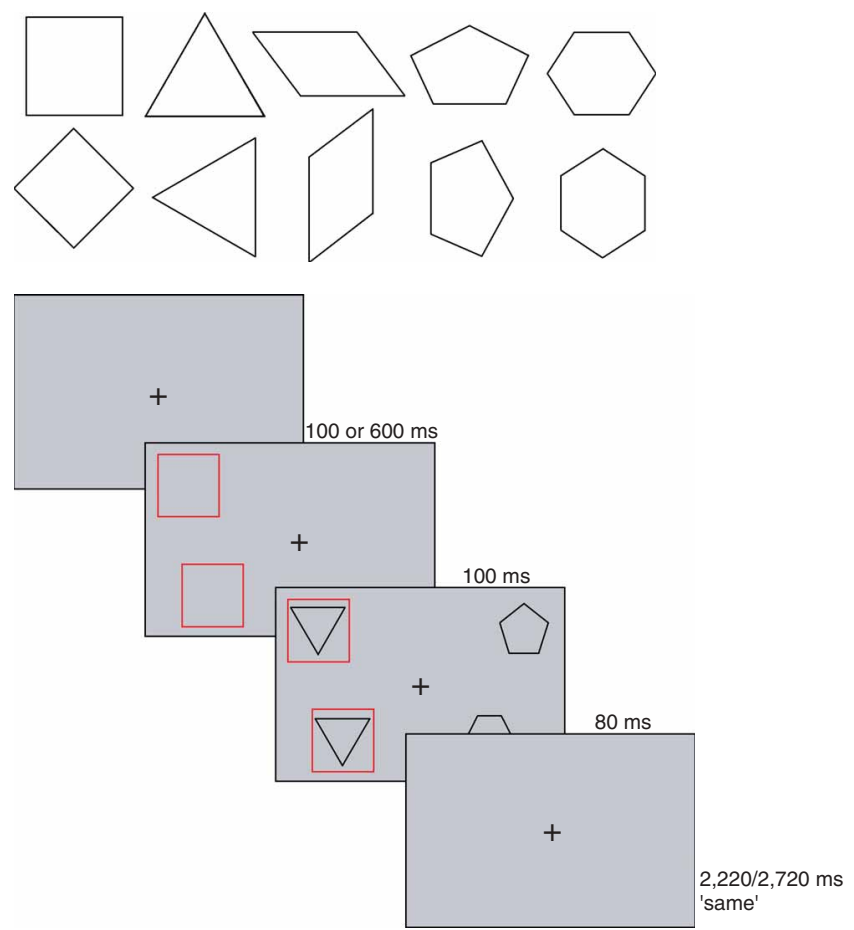

${ }^{1}$ Department of Experimental Psychology, Otto von Guericke University, Postbox 4120, 39016 Magdeburg, Germany. ${ }^{2}$ Day Clinic of Cognitive Neurology, University of Leipzig, Liebigstrasse 22a, 04103 Leipzig, Germany. ${ }^{3}$ Department of Cognitive Neurology, Max Planck Institute for Human Cognitive and Brain Sciences, Stephanstrasse 1a, 04103 Leipzig, Germany. Correspondence should be addressed to S.P. (stefan.pollmann@nat.uni-magdeburg.de). 
a
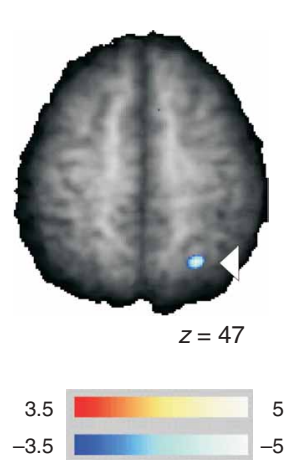

b

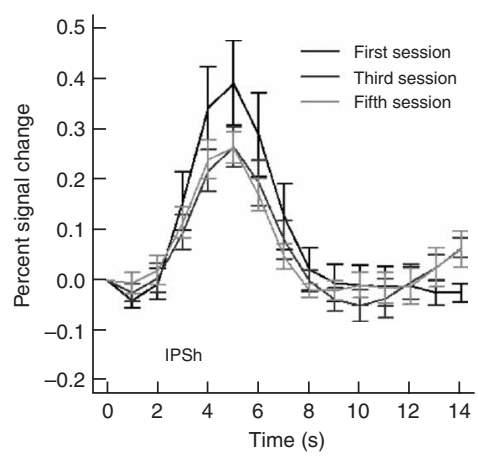

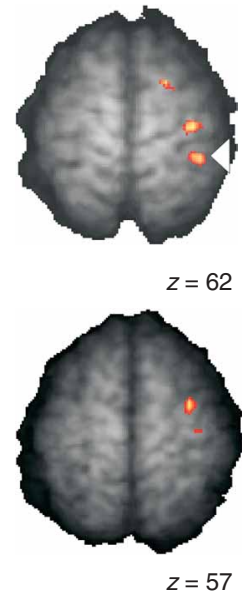

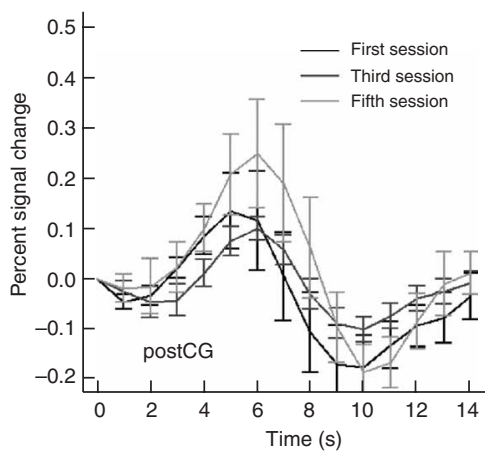

Figure 2 Learning-related activation changes. The figure shows the activation changes between sessions 5 and 1 . The red-yellow scale shows signal increase over sessions, whereas the blue scale shows signal decrease (Z-scores). Left hemisphere is on the left. (a) Activation in the horizontal segment of the intraparietal sulcus (IPSh, Talairach coordinates 25, -64, 47). (b) Activation in postcentral gyrus (postCG, 31, -36, 62) bordering the superior postcentral sulcus (arrow), and local activation maxima in precentral gyrus and superior frontal gyrus shown in two axial planes. $z, z$-coordinate in Talairach space. The graphs indicate average signal time courses (mean \pm s.e.m.) in sessions 1,3 and 5.

In our visual object matching task, we expected that, during the course of learning, activation would decrease along the horizontal segment of the IPS, as would the need to attentively process individual object features. By contrast, we expected activation to increase in the motor and sensory hand areas and in dorsal premotor cortex as objectresponse associations were strengthened.

To test these predictions, we used a geometric figure matching task (Fig. 1). Geometric objects were chosen because they are familiar (but not as overlearned as letters); thus object matching was initially based on feature comparisons. Stimuli were presented tachistoscopically to prevent eye movements. Participants were asked to judge common geometric figures by either their physical (PI) or their categorical (CI) identity. They were required to indicate matches and non-matches by a forced choice response, and a feedback tone indicated an incorrect response; thus a consistent association between object pairs and correct responses could be learned in the course of the experiment. Participants took part in five sessions over a period of up to $21 \mathrm{~d}$. fMRI data were collected in the first, third and fifth sessions (see Supplementary Methods). Participants considerably improved their performance on the task, as indicated by a decrease in response latency from the first session $(757 \mathrm{~ms})$ to the third $(694 \mathrm{~ms})\left(t_{6}=4.62 ; P<0.05\right)$ and from the third session to the fifth $(651 \mathrm{~ms})\left(t_{6}=3.22 ; P<0.05\right)$. Likewise, error rates declined from $8.3 \%$ in the first session to $4.5 \%$ in the third and $2.8 \%$ in the final session.

In agreement with our hypothesis, we observed a reduction in activation between the first and last fMRI sessions (equivalent to the fifth training session) along the banks of the horizontal segment ${ }^{5}$ of the right intraparietal sulcus (Fig. 2a). Notably, the reverse (that is, an increase in activation between sessions 1 and 5) was observed in the right precentral gyrus, extending anteriorly into the superior frontal gyrus and posteriorly into the postcentral gyrus. Within these large activated areas, local activation maxima were observed in the middle genu of the precentral gyrus (the motor hand area; ref. 11), posteriorly adjacent in the somatosensory hand representation area in postcentral gyrus, further anterior at the banks of the superior precentral sulcus (dorsal premotor cortex; ref. 10) and in the posterior portion of the superior frontal gyrus (Fig. 2b, Supplementary Table 1).
In the right postcentral gyrus, there was a strong increase in signal amplitude in session 5 as compared to sessions 1 and 3 (Fig. 2b); these last did not differ from each other in signal amplitude. This pattern was observed for trials requiring left hand and right hand responses alike (Supplementary Fig. 1); thus, the signal increase over sessions did not reflect processes related to contralateral motor execution. In contrast, we observed a decrease in signal amplitude between session 1 and sessions 3 and 5 (with no differences between the last two) along the horizontal segment of the right intraparietal sulcus (Fig. 2a). These learning-related activation changes were comparable for physical and category matching (Supplementary Fig. 2); this shows that the activations were not associated with task-specific operations such as mental rotation (a potentially useful strategy in the CI, but not the PI, task).

We did not observe learning-related signal changes in visual object processing areas in the lateral occipital cortex or the fusiform gyrus (the lateral occipital complex ${ }^{12}$ ). This may be due to the fact that no novel object representation needed to be generated (as subjects were familiar with the geometric objects used in this study); rather, an association between existing object representations needed to be built.

We have hypothesized that in the present study, training should lead to memory-based processing, thus reducing the demands on attentive processing. The decrease in activation along the horizontal segment of the right intraparietal sulcus, at a location consistently reported to subserve attentive processing, confirms the first part of our hypothesis. More importantly, we have posited that learning object matching includes associating object pairs with the appropriate response. The signal increase over sessions in the hand representation areas in precentral and postcentral gyri supports this prediction. It may seem puzzling that a simple two-alternative forced-choice response should require learning. However, in our view, it is not the response that needs to be learned but the link between a particular object pair and its associated response (which is strengthened when the same object pair is presented repeatedly and is followed by the same response). Postcentral gyrus activation, that is, activation in sensorimotor cortex (S1), is commonly observed in motor tasks, often with greater signal changes 


\section{BRIEF COMMUNICATIONS}

than in precentral motor areas ${ }^{13}$. The rostral part of the dorsal premotor cortex, along the banks of the superior precentral sulcus, and the cortex along the horizontal segment of the IPS - both activated in the present study-have previously been reported to support conditional motor selection ${ }^{10}$.

Our data agree well with these data; moreover, they show that the contributions of the posterior parietal and more anterior components can be further dissociated in the time course of establishing a link between object representation and action. The early decrease in activation along the IPS (between sessions 1 and 3) precedes the later increase in activation in the postcentral gyrus (between sessions 3 and 5). This shows that the latter is not immediately contingent on the former; it may indicate that learning in the object matching task occurred in two steps: an initial step in which a perceptual representation of the object pair was generated, reducing visual attentional demands, and a second step in which the perceptual object representation was associated with the appropriate response 4 . According to such a sequential model, fast learners may have begun responserelated learning even before session 3, whereas slow learners may reach this transition only after session 3. Indeed, the increase in postcentral signal between sessions 3 and 5 was significantly correlated with the ratio of late to early response-time reductions $(r=0.784, P<0.05$; Supplementary Fig. 3); the signal increase was stronger for late learners. However, the relation between perceptual and response learning may depend on numerous factors, such as object complexity or salience on the perceptual learning side and the number of response alternatives on the response learning side. Furthermore, perceptual learning can proceed much faster than in the present experiment and the contribution of parietal cortex may decrease rapidly ${ }^{14}$. Are object-response associations created equally fast under these circumstances? Longitudinal fMRI studies may be a valuable tool to address these issues.
In summary, the decrease in activation in the posterior part of the IPS, along with an increase in activation in the postcentral gyrus and the frontal cortex, support the idea that learning object matching proceeds from an initial attentionally demanding feature comparisons stage to a later template-based processing stage in which object pairs are linked to the appropriate response.

Note: Supplementary information is available on the Nature Neuroscience website.

\section{ACKNOWLEDGMENTS}

This work was supported by a grant from the Gertrud Reemtsma Stiftung to M.M. and by Deutsche Forschungsgemeinschaft grant Po 548/3-1 to S.P.

\section{COMPETING INTERESTS STATEMENT}

The authors declare that they have no competing financial interests.

Published online at http://www.nature.com/natureneuroscience/

Reprints and permissions information is available online at http://npg.nature.com/ reprintsandpermissions/

1. Hommel, B. Vis. Cogn. 5, 183-216 (1998).

2. Logan, G.D. Psychol. Rev. 109, 376-400 (2002).

3. Hommel, B., Musseler, J., Aschersleben, G. \& Prinz, W. Behav. Brain Sci. 24, 849-878 (2001).

4. Walsh, V., Ashbridge, E. \& Cowey, A. Neuropsychologia 36, 45-49 (1998).

5. Duvernoy, H.M. The Human Brain: Surface, Three-Dimensional Sectional Anatomy with MRI, and Blood Supply 2nd edn. (Springer-Verlag, New York, 1999).

6. Corbetta, M. et al. Neuron 21, 761-773 (1998).

7. Vandenberghe, R., Gitelman, D.R., Parrish, T.B. \& Mesulam, M.M. Neuroimage 14, 661-673 (2001).

8. Weidner, R., Pollmann, S., Müller, H.J. \& von Cramon, D.Y. Cereb. Cortex 12, 318-328 (2002).

9. Poldrack, R.A., Desmond, J.E., Glover, G.H. \& Gabrieli, J.D.E. Cereb. Cortex 8, 1-10 (1998).

10. Grafton, S.T., Fagg, A.H. \& Arbib, M.A. J. Neurophysiol. 79, 1092-1097 (1998).

11. Yousry, T.A. et al. Brain 120, 141-157 (1997).

12. Malach, R., Levy, I. \& Hasson, U. Trends Cogn. Sci. 6, 176-184 (2002).

13. Maldjian, J.A., Gottschalk, A., Patel, R.S., Detre, J.A. \& Alsop, D.C. Neuroimage 10, 55-62 (1999).

14. Walsh, V., Allison, A., Ashbridge, E. \& Cowey, A. Neuropsychologia 37, 245-251 (1999). 